\title{
Hydrogenation of Semiconductor Surfaces: Si and Ge (111)
}

\section{Citation}

Kaxiras, Efthimios and John D. Joannopoulos. 1988. Hydrogenation of semiconductor surfaces: Si and Ge (111). Physical Review B 37(15): 8842-8848.

\section{Published Version}

http://dx.doi.org/10.1103/PhysRevB.37.8842

\section{Permanent link}

http://nrs.harvard.edu/urn-3:HUL.InstRepos:2961255

\section{Terms of Use}

This article was downloaded from Harvard University's DASH repository, and is made available under the terms and conditions applicable to Other Posted Material, as set forth at http:// nrs.harvard.edu/urn-3:HUL.InstRepos:dash.current.terms-of-use\#LAA

\section{Share Your Story}

The Harvard community has made this article openly available.

Please share how this access benefits you. Submit a story.

\section{Accessibility}




\title{
Hydrogenation of semiconductor surfaces: Si and Ge (111)
}

\author{
E. Kaxiras and J. D. Joannopoulos \\ Department of Physics, Massachusetts Institute of Technology, Cambridge, Massachusetts 02139
}

(Received 27 February 1987; revised manuscript received 1 June 1987)

\begin{abstract}
The relaxations of hydrogenated $\mathrm{Si}$ and $\mathrm{Ge}(111)$ surfaces are determined using ab initio selfconsistent calculations in a slab configuration. The $\mathrm{Si}-\mathrm{H}$ and $\mathrm{Ge}-\mathrm{H}$ bonds are found to be considerably larger than the sum of covalent radii. The substrate relaxations are small and their physical origin can be explained in terms of electronic charge transfer which eliminates the surface dipole moment. The calculated frequencies of the hydrogen vibrational modes are in excellent agreement with experiment. A surface-atom vibrational mode is compared to similar modes in the amorphous hydrogenated materials. The comparison predicts that internal surfaces (microvoids) in the amorphous network are locally much softer than the corresponding crystalline surface configuration.
\end{abstract}

The interaction of atomic hydrogen with cleaved semiconductor surfaces has been extensively studied for over a decade. The earliest experimental work ${ }^{1-3}$ and theoretical calculations ${ }^{4-7}$ confirmed the basic, straightforward interpretation of hydrogenation in the model system considered, namely Si(111). Specifically, hydrogen atoms saturate the surface dangling bonds, giving a nearly ideal, bulk-terminated plane of exposed surface atoms. It is interesting that in cases where the surface does not have the geometry and periodicity of the bulk-terminated plane, the interaction of hydrogen with surface atoms is strong enough to unreconstruct the complicated reconstruction patterns. This process takes place, for example, on the $(2 \times 1) \mathrm{Si}(111)$ surface, which exhibits a low-energy $\pi$-bonded-chain reconstruction. ${ }^{8}$ Upon hydrogenation this chain of $\mathrm{Si}$ atoms with $(2 \times 1)$ periodicity reverts to the $(1 \times 1)$ pattern of the bulk-terminated plane. Similar phenomena have been observed on the Ge(111) surface. ${ }^{9}$ More recent experimental ${ }^{10,11}$ and theoretical ${ }^{12,13}$ work has concentrated on determining vibrational frequencies of the hydrogenated surfaces. However, a first-principles study with adequate accuracy to determine these frequencies is lacking.

Despite their apparent simplicity, these nearly ideal surfaces are important physical systems on which detailed investigations can be performed leading to better understanding of other, more complicated but structurally related systems. For example, the hydrogenation of amorphous $\mathrm{Si}$, a process of particular technological importance, exhibits, at some level, characteristics comparable to the hydrogenation of crystalline surfaces.

The purpose of the present paper is twofold. First, to show, through detailed $a b$ initio quantum-mechanical calculations, that the atomic positions of the hydrogenated $\mathrm{Si}$ and $\mathrm{Ge}$ (111) surfaces differ significantly from those of a bulk-terminated plane. Previous theoretical work has not addressed this issue, either because it was considered unimportant to the reported results (which is probably true in interpreting photoemission measurements), or due to the inability of the method to calculate accurately the relaxation of the substrate: for example, in cluster models with up to $10 \mathrm{Si}$ or Ge atoms, ${ }^{12,13}$ all of the atoms are surface atoms, and the substrate influence on surface reconstruction is not properly taken into account. Another shortcoming of the cluster models is the lack of long-range order, which is present in the real system and can, in principle, alter the relaxation observed, due to different correlations in the electronic wave functions.

The second purpose of the paper is to give an example of how the calculated structural parameters of the hydrogenated surfaces can give insight to the microscopic atomic configurations of the amorphous material.

In order to obtain reliable values for the surface relaxation we choose to model the system by a slab consisting of eight atomic planes (prior to hydrogenation) in the $\langle 111\rangle$ direction. The top two layers of each side of the slab are allowed to relax fully. The slabs are separated by a vacuum distance equal to about four times the crystal bond length. The atoms are modeled by the nonlocal norm-con'serving pseudopotentials of Bachelet, Hamann, and Schlüter. ${ }^{14}$ The total energy of the different atomic configurations was minimized to obtain the relaxation. Total energies were calculated using the self-consistent formalism of Ihm, Zunger, and Cohen, ${ }^{15}$ with the Ceperly-Alder approximation ${ }^{16}$ for the exchangecorrelation energy as parametrized by Perdew and Zunger. ${ }^{17}$ The wave functions were expanded in a planewave basis which consists of plane waves with kinetic energy up to $10 \mathrm{Ry}$. An additional set of plane waves with energy up to $16 \mathrm{Ry}$ was taken into account by secondorder perturbation theory. ${ }^{18}$ The plane-wave basis corresponds to $\sim 100$ plane waves per atom with an additional $\sim 100$ plane waves per atom included through perturbation theory. These cutoffs are sufficient to reproduce accurately relaxation energies of the order of a few meV. ${ }^{19}$ The relative accuracy for energies of slightly distorted structures in the present calculation is $\sim 10^{-4} \mathrm{eV}$. Four points in the surface Brillouin zone were used to estimate averages over the entire zone. They were chosen using the Monkhorst-Pack scheme, ${ }^{20}$ and were shifted to highsymmetry points to facilitate the computation.

Three relaxation parameters were considered and varied independently to obtain the optimal configuration: the length of the hydrogen bond $\left(b_{H}\right)$, the first-to-second 
layer spacing $\left(d_{12}\right)$ and the second-to-third layer spacing $\left(d_{23}\right)$. These are illustrated in Fig. 1 and the values obtained are summarized in Table $\mathrm{I}$. Surprisingly, the $\mathrm{Si}-$ $\mathrm{H}$ bond is found to be $1.54 \AA$, which is considerably larger than the value obtained by adding the atomic covalent radii ${ }^{21}(1.48 \AA)$. The latter value has been used in previous calculations ${ }^{4,7,22}$ since it is equal to the experimental value for the $\mathrm{Si}-\mathrm{H}$ bond in $\mathrm{SiH}_{4}$. The chemical environment of the (111) surface, however, is definitely different from that of $\mathrm{SiH}_{4}$ and the larger bond length on the surface is a direct consequence of this difference (as will be explained below). Similar remarks hold for $\mathrm{Ge}$, although here the deviation of the calculated $\mathrm{Ge}-\mathrm{H}$ bond $(1.57 \AA$ ) from the sum of the covalent radii $(1.53 \AA)$ is not as large.

We consider next the relaxation of the surface layer spacings $d_{12}$ and $d_{23}$. In both cases there is a contraction with respect to the ideal spacings, which alters the relative bonding configuration of the surface atoms. The bond lengths and bond angles tabulated in Table I give a measure of the distortion with respect to the ideal diamondlike values. This relaxation is probably hard to determine experimentally, because the deviation from the ideal structure is so small. Only very recently a lowenergy electron diffraction (LEED) experiment has succeeded in determining values for the relaxation of $d_{12}$ and $d_{23}$ in $\mathrm{Ge}(111): \mathrm{H}^{23}$ The LEED-determined relaxation for $d_{12}\left(\Delta d_{12}=-0.10 \AA\right)$ is in good agreement with the value obtained from Table $\mathrm{I}\left(\Delta d_{12}=-0.08 \AA\right.$ ), whereas that for $d_{23}$ is opposite in sign $\left(\Delta d_{23}=+0.05 \AA\right)$ than what we calculate $\left(\Delta d_{23}=-0.07 \AA\right)$. The LEED analysis used a shorter bond length for $\mathrm{Ge}-\mathrm{H}$ than our value $(1.57 \AA$ ) and it remains to be seen whether this is the reason for the discrepancy in the $d_{23}$ relaxations. The computational scheme employed here reproduces experimental bulk bond distances typically to $\sim 1 \%,{ }^{24}$ thus the uncertainty in the bond relaxations quoted above versus experimental values is of the order of $0.02 \AA$.

For purposes of comparison, we also calculated the re-

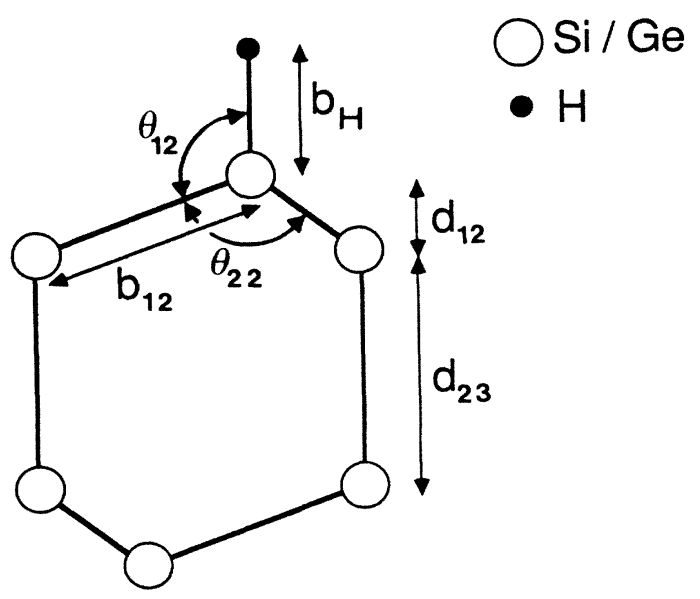

FIG. 1. Side view of the (111) surface of $\mathrm{Si}$ and Ge. The bond lengths, interplanar distances, and bond angles discussed in the text are defined.
TABLE I. The calculated relaxations of the hydrogenated $\mathrm{Si}$ and $\mathrm{Ge}(111)$ surfaces. The parameters $b_{\mathrm{H}}, d_{12}, d_{23}, \theta_{12}, \theta_{22}$, and $b_{12}$ are defined in Fig. 1. All lengths are given in $\AA$. $(\mathbf{H})$ and (clean) refer to the hydrogenated and unhydrogenated surfaces. The ideal values correspond to unrelaxed diamond-lattice configuration.

\begin{tabular}{lrccc}
\hline & \multicolumn{2}{c}{$\mathrm{Si}$} & \multicolumn{2}{c}{$\mathrm{Ge}$} \\
& Relaxed & Ideal & Relaxed & Ideal \\
\hline$b_{\mathrm{H}}$ & 1.54 & $1.48^{\mathrm{a}}$ & 1.57 & $1.53^{\mathrm{a}}$ \\
$d_{12}(\mathbf{H})$ & 0.72 & 0.78 & 0.73 & 0.81 \\
$d_{12}$ (clean) & 0.57 & 0.78 & 0.30 & 0.81 \\
$d_{23}$ & 2.32 & 2.35 & 2.38 & 2.45 \\
$b_{12}$ & 2.33 & 2.35 & 2.42 & 2.45 \\
$\theta_{12}$ & $108.1^{\circ}$ & $109.5^{\circ}$ & $107.7^{\circ}$ & $109.5^{\circ}$ \\
$\theta_{12}$ & $110.8^{\circ}$ & $109.5^{\circ}$ & $111.2^{\circ}$ & $109.5^{\circ}$ \\
\hline \hline
\end{tabular}

${ }^{\text {a }}$ Sum of covalent radii (Ref. 21 ).

laxation of the first bilayer spacing for a clean, unhydrogenated $(111)(1 \times 1)$ surface of $\mathrm{Si}$ and $\mathrm{Ge}$. Although this configuration may not be actually realized (because of reconstruction of the clean surface) the relaxation associated with it gives an indication of how hydrogenation affects the surface. Indeed, the change in the first bilayer spacing for the clean surfaces is several times larger than the change in the hydrogenated surfaces (approximately five times larger for $\mathrm{Si}$ and about six times for $\mathrm{Ge}$ ).

Since the adsorption of $\mathbf{H}$ completely saturates the surface dangling bonds, the existence of residual substrate relaxation remains a puzzling question. In order to explain the relaxation we consider the difference in electronegativity between the $\mathrm{Si}$ and $\mathrm{H}$ atoms. ${ }^{21}$ This electronegativity difference, in the absence of any relaxation (with the $\mathrm{Si}-\mathrm{H}$ bond length equal to the sum of covalent radii) will produce a dipole moment in the surface. An elongation of the $\mathrm{Si}-\mathrm{H}$ bond and contraction of the surface $\mathrm{Si}$ back bonds leads to electronic charge transfer from the hydrogen bond to the back bonds. This firstorder relaxation polarizes the electron distribution in such a way as to partially cancel the dipole moment. A second-order relaxation effect is the contraction of the second-to-third layer spacing which completes the cancellation of the dipole moment by spreading the charge transferred from the hydrogen bond over a larger region of substrate bonds. Similar comments apply to the relaxation of the Ge surface, since the electronegativity of $\mathrm{Ge}$ is the same as that of $\mathrm{Si}^{21}$

The above argument explains in simple physical terms both the substrate relaxation and the $\mathrm{Si}-\mathrm{H}$ or $\mathrm{Ge}-\mathrm{H}$ bond elongation. In order to substantiate this qualitative argument we show the total valence charge density for $\mathrm{Si}(111): \mathrm{H}$ and $\mathrm{Ge}(111): \mathrm{H}$ in Figs. 2 and 3, respectively, in the ideal and the fully relaxed configurations. The plane of these figures is the (110) plane of the diamond crystal, with the familiar chains of $\mathrm{Si}$ or $\mathrm{Ge}$ atoms (open circles) terminated by $\mathbf{H}$ atoms (solid circles) at the surface. The difference between the ideal and relaxed configurations is also shown for each case, with positive charge contours (regions to which charge was added) in continuous lines, and negative charge contours (regions from which charge 

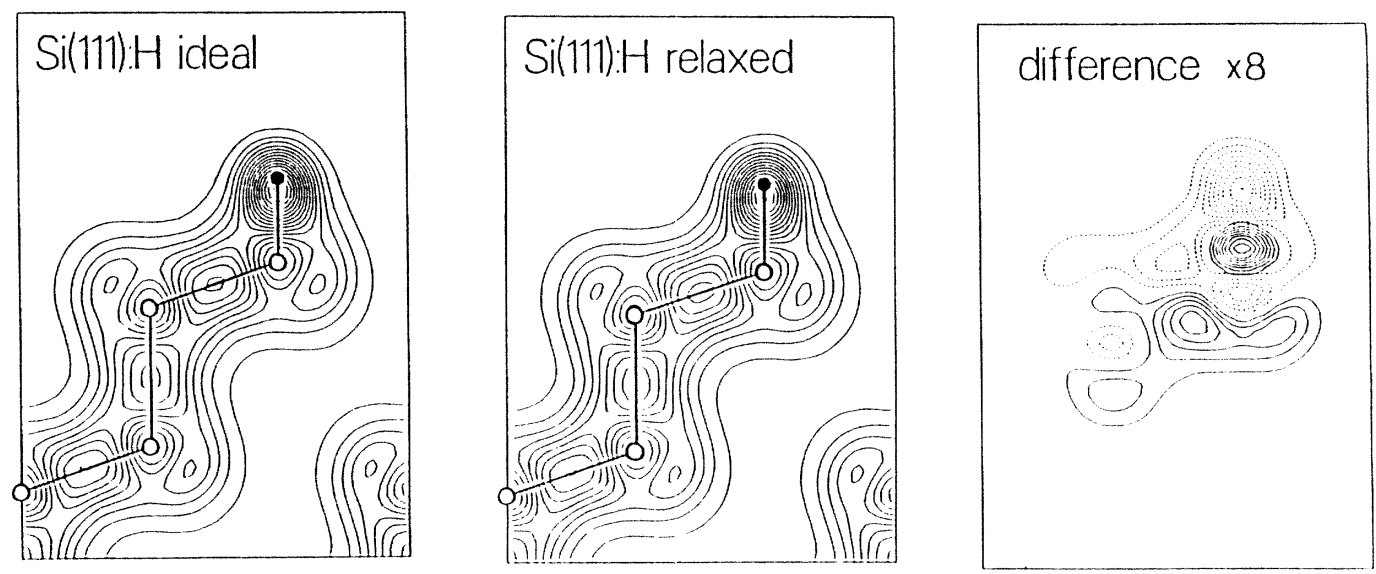

FIG. 2. Valence charge density of $\mathrm{Si}(111): \mathrm{H}$ on the (110) plane. Open circles correspond to $\mathrm{Si}$ atoms and solid circles to $\mathrm{H}$ atoms. The contour spacing is in units of $\frac{4}{3} e^{-}$per bulk atomic value. The difference between the ideal and fully relaxed configurations is amplified by a factor of 8 (contour spacing is $\frac{1}{6} e^{-}$per bulk atomic volume). Positive contours are shown in continuous lines, negative ones in dotted lines.

was subtracted) in dotted lines. Apart from a charge shift due to the downward displacement of the $\mathrm{Si}-\mathrm{H}$ or $\mathrm{Ge}-$ $\mathbf{H}$ bond, the charge transfer takes place in exactly the manner described in the qualitative argument above.

We turn now to the determination of vibrational frequencies from the total-energy curves. There are two vibrational modes that are easy to identify in terms of atomic motions; they both involve hydrogen atoms moving relative to the substrate. The large difference in mass between $\mathrm{H}$ and $\mathrm{Si}$ and $\mathrm{Ge}$ effectively decouples the motion of $\mathbf{H}$ from collective modes of the lattice. The two different modes involve stretching (frequency $\omega_{\mathbf{H}}^{\text {stretch }}$ ) and bending (also called wagging; frequency $\omega_{\mathrm{H}}^{\text {wag }}$ ) of the $\mathrm{Si}-\mathrm{H}$ and $\mathrm{Ge}-\mathrm{H}$ bonds. For these vibrational modes there exist several experimental measurements. ${ }^{11,25,26}$

Table II summarizes the theoretical estimates for $b_{H}$ and $\omega_{\mathrm{H}}^{\text {stretch }}$ as given by different authors. It is seen that the values of $b_{H}$ vary considerably, with the cluster calculations (Refs. 12 and 13) giving shorter bond lengths than the self-consistent slab calculations (Ref. 7 and present work). An interesting question is whether the proper relaxation of the surface significantly affects the vibrational frequencies. In order to investigate this we calculated the frequency $\omega_{\mathrm{H}}^{\text {stretch }}$ in $\mathrm{Si}(111): \mathrm{H}$ and $\mathrm{Ge}(111): \mathrm{H}$ for different relaxations, by fitting quadratic polynomials to the total energy values. In Fig. 4 we show an example of the calculated energies and corresponding fitted curves for the Si-H [Fig. 4(a)] and Ge-H [Fig. 4(b)] bond stretching modes in the fully relaxed configuration. Higher-order polynomial fits give frequencies that differ by less than $1 \%$ from the quadratic-fit values, for all the cases considered. The almost perfect harmonic character of the energy curves is a reflection of the high degree of convergence of the calculations, which gives the energy differences with a relative accuracy of $10^{-4} \mathrm{eV}$, as mentioned earlier.

Table III lists the values of the frequency $\omega_{\mathrm{H}}^{\text {stretch }}$ for three different relaxations: first without any relaxation of the first two layers, second with full relaxation of the first layer only, and finally with full relaxation of the first and second layers. The corresponding equilibrium value of the $\mathrm{Si}-\mathrm{H}$ and $\mathrm{Ge}-\mathrm{H}$ bonds is also given, as well as the
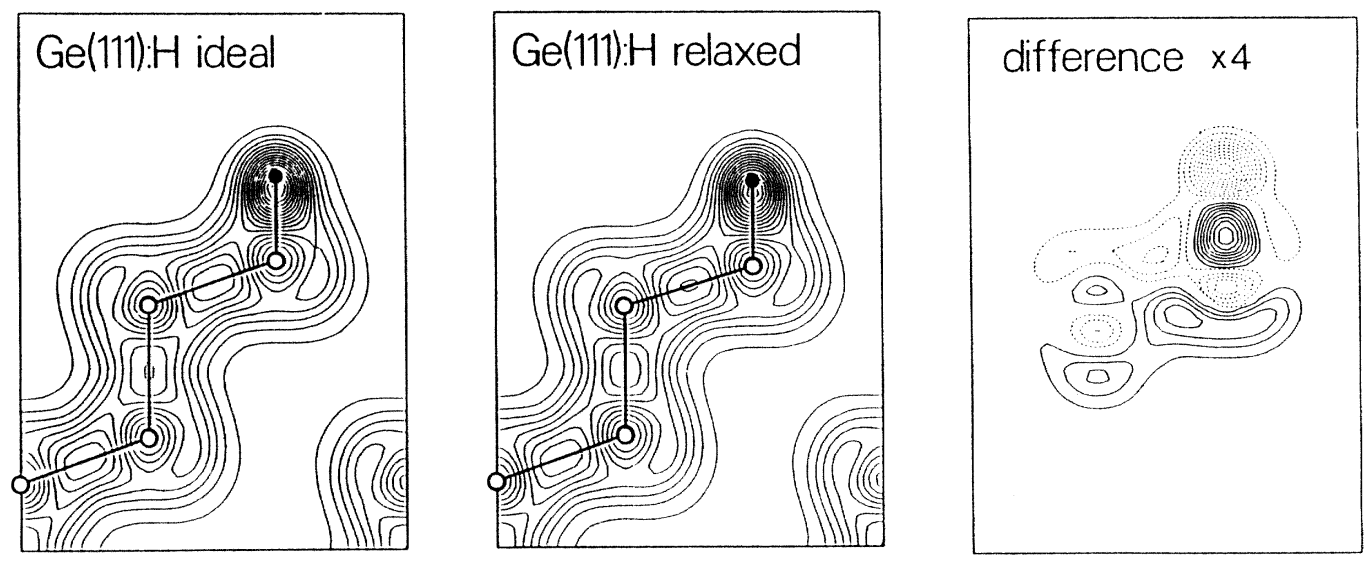

FIG. 3. Same as in Fig. 2, for the Ge(111):H surface. The difference contours are amplified by a factor of 4. 
TABLE II. Comparison of theoretical values for the $\mathrm{Si}-\mathrm{H}$ bond length $\left(b_{\mathrm{H}}\right.$, in $\AA$ ) and the hydrogen stretching mode ( $\omega_{\mathrm{H}}^{\text {stretch }}$, in $\mathrm{meV}$ ) on the $\mathrm{Si}(111): \mathrm{H}$ surface, as calculated by different authors.

\begin{tabular}{lccccc}
\hline \hline & Ref. 4 & Ref. 7 & Ref. 12 & Ref. 13 & This work \\
\hline$b_{\mathrm{H}}(\AA)$ & 1.445 & 1.550 & 1.486 & 1.484 & 1.544 \\
$\omega_{\mathrm{H}}^{\text {stretch }}(\mathrm{meV})$ & 270 & & 282 & 271 & 245 \\
\hline \hline
\end{tabular}

${ }^{a}$ Values from the largest cluster used in Ref. 12.

b Values from the largest basis set used in Ref. 13.

total relaxation energy in $\mathrm{eV}$ per $(1 \times 1)$ surface unit cell. It is interesting that the $\mathrm{Si}-\mathrm{H}$ and $\mathrm{Ge}-\mathrm{H}$ bonds are constant to within $1 \times 10^{-3} \AA$. The vibrational frequencies, however, vary by a small but non-negligible amount: a comparison of their values shows that the first layer relaxation produces considerable softening of the $\mathrm{Si}-\mathrm{H}$ and $\mathrm{Ge}-\mathrm{H}$ stretching modes, while the second layer relaxation stiffens the modes by a smaller amount. It should be pointed out that no relative errors are introduced in this comparison since the level of accuracy for all the energies and the fitting procedure are the same for
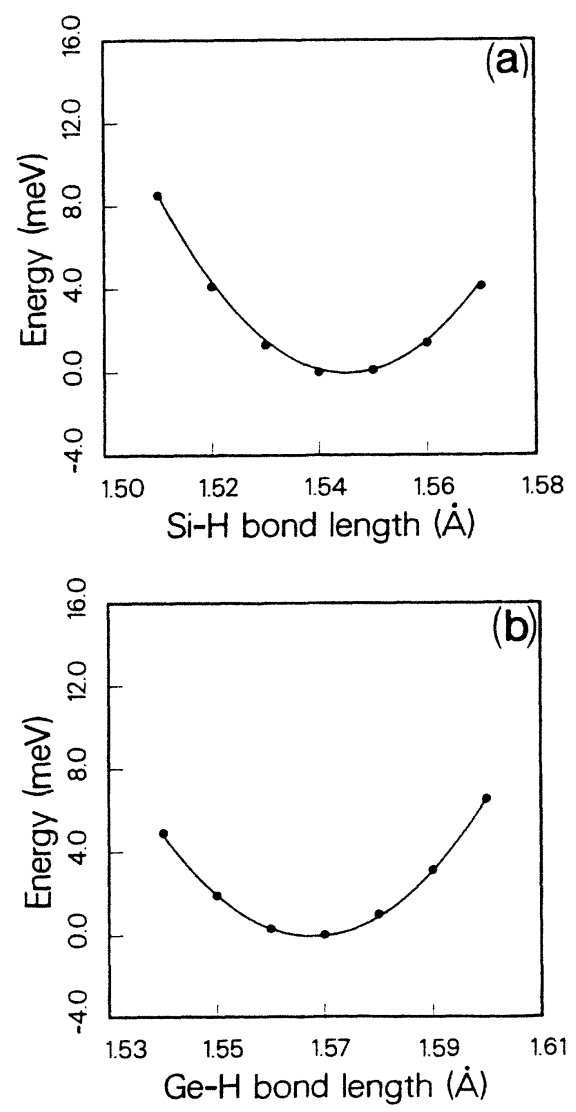

FIG. 4. Calculated total energies and quadratic polynomial fits for the hydrogen bond stretching mode in (a) $\mathrm{Si}(111): \mathrm{H}$ and (b) $\mathrm{Ge}(111): \mathrm{H}$. Energies are given in $\mathrm{meV}$ per $(1 \times 1)$ surface unit cell, with respect to the fully relaxed configuration. all the relaxations; the systematic errors therefore do not affect the discussion of the relative stiffness. The frequencies at full relaxation differ from the experimental value by $-5 \%$ for $\mathrm{Si}$ and by $-6 \%$ for $\mathrm{Ge}$, which is the typical accuracy expected from the computational scheme for the curvature of total-energy curves ${ }^{24}$ (e.g., bulk moduli).

We have also calculated the frequency of the wagging modes $\left(\omega_{\mathrm{H}}^{\mathrm{Wag}}\right)$ and the frequency for vibration of the top layer $\left(\omega_{1}\right)$. The corresponding energy curves are shown in Figs. 5 and 6, respectively [Figs. 5(a) and 6(a) for $\mathrm{Si}$, Figs. 5(b) and 6(b) for Ge]. The frequencies for the three modes $\left(\omega_{\mathbf{H}}^{\text {stretch }}, \omega_{\mathbf{H}}^{\text {wag }}\right.$, and $\left.\omega_{1}\right)$ and the experimental values are collected in Table IV. There is no experimental measurement for $\omega_{1}$ because it lies in the region of other vibrational modes of the crystal and couples strongly to them. There is, however, a similar vibrational mode in the amorphous materials for which experimental measurements exist. ${ }^{27}$ Vibrational frequencies associated with $\mathrm{H}$ motion in amorphous hydrogenated $\mathrm{Si}$ and $\mathrm{Ge}$ have also been determined experimentally ${ }^{28,29}$ and their values are included in Table IV.

The calculated values for $\omega_{\mathrm{H}}^{\text {stretch }}$ and $\omega_{\mathrm{H}}^{\text {wag }}$ are in excellent agreement with the experimentally determined frequencies. By contrast the calculated values for $\omega_{1}$ differ by $\sim 35 \%$ from the experimental ones. Given the good agreement we can obtain between theory and experiment for the hydrogen-related vibrations, we conclude that the discrepancy in the values of $\omega_{1}$ is not related to some theoretical shortcoming. In particular, the systematic errors in the hydrogen relaxations and the surface layer relaxations are the same and the expected accuracy for the $\omega_{1}$ mode is therefore $5-10 \%$. The large discrepancy in $\omega_{1}$ indicates a significant difference between the motion of atoms in the hydrogenated surfaces and in microvoids of the amorphous material.

Let us consider then in more detail the physics of this particular vibrational mode. Although different interpretations of the atomic motions have been proposed, ${ }^{27,30,31}$ we will assume (based on the arguments of Martinez and Yndurain $^{31}$ and of Cardona ${ }^{32}$ ) that the most plausible model involves the motion of a singly hydrogenated $\mathrm{Si}$ atom. Our calculations for the hydrogen modes underestimate the experimental frequencies by $\sim 5 \%$. Thus, assuming the same level of theoretical uncertainty in $\omega_{1}$, we see that the frequency of this vibrational mode is roughly $40 \%$ higher in crystalline surfaces than in amorphous microvoids. One obvious mechanism which would decrease this frequency is a concerted motion of the singly hydrogenated $\mathrm{Si}$ atom with its neighbors. To first order, this 
TABLE III. Variation of the hydrogen stretching mode ( $\left.\omega_{\mathrm{H}}^{\text {stretch }}\right)$ with surface relaxation for $\mathrm{Si}(111): \mathrm{H}$ and $\mathrm{Ge}(111): \mathrm{H}$. The relaxation is defined by the deviations of the first-to-second layer $\left(\Delta d_{12}\right)$ and the second-to-third layer $\left(\Delta d_{23}\right)$ spacings from their ideal values. The total energy of the relaxation $(\Delta E)$ is also given in $\mathrm{eV}$ per $(1 \times 1)$ surface unit cell, as well as the equilibrium bond length of the hydrogen bond $\left(b_{H}\right)$. Frequencies are in $\mathrm{meV}$ and lengths in $\AA$.

\begin{tabular}{llccccc}
\hline & \multicolumn{3}{c}{$\mathrm{Si}$} & \multicolumn{3}{c}{$\mathrm{Ge}$} \\
\hline$\Delta d_{12}$ & 0.00 & -0.06 & -0.06 & 0.00 & -0.08 & -0.08 \\
$\Delta d_{23}$ & 0.00 & 0.00 & -0.03 & 0.00 & 0.00 & -0.07 \\
$\Delta E$ & 0.000 & -0.016 & -0.022 & 0.000 & -0.026 & -0.043 \\
$b_{\mathrm{H}}$ & 1.542 & 1.543 & 1.544 & 1.567 & 1.567 & 1.568 \\
$\omega_{\mathrm{H}}^{\text {stretch }}$ & 257.6 & 239.9 & 245.3 & 237.0 & 229.3 & 230.6 \\
\hline \hline
\end{tabular}

concerted motion will include only nearest neighbors and will give rise to two modes: the center of mass of the three nearest neighbors can move in unison with the $\mathrm{Si}-\mathrm{H}$ complex (symmetric mode) or against it (antisymmetric mode). Both modes should be infrared active due to the symmetry-breaking hydrogen bond. ${ }^{33}$

A theoretical model based on the Bethe-lattice approximation has shown that it is actually the antisymmetric mode which corresponds to the experimentally observed sharp peak in the infrared absorption spectrum. ${ }^{31}$ Such a mode, however, will have a frequency even higher than the calculated value of $\omega_{1}$. Consequently, we conclude that the force constants in the amorphous microvoid are considerably smaller than the corresponding configuration on the (111) surface. Since the value of $\omega_{1}$ for $\mathrm{Si}(111): \mathrm{H}$ is $40 \%$ larger than that for $\alpha-\mathrm{Si}: \mathrm{H}$, we expect the force constants (proportional to $\omega^{2}$ ) to be smaller by a factor of 2 in the amorphous material. Bond lengths in amorphous $\mathrm{Si}$ are similar to the crystalline values to within $\sim 1 \%$, whereas bond angles deviate from the tetrahedral value by $\sim 10 \%$. This leads us to suggest that the major part of the discrepancy in the forces discussed above is due to differences in angular force constants: they have to be weaker in internal surfaces of the amor-
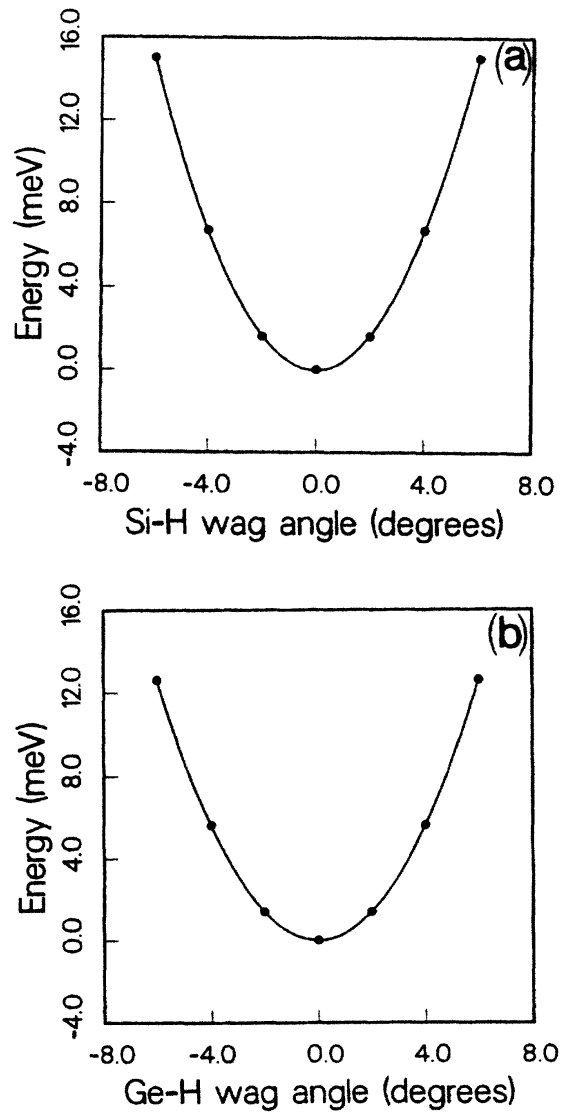

FIG. 5. Same as in Fig. 4 for the hydrogen bond wagging (bending) mode.
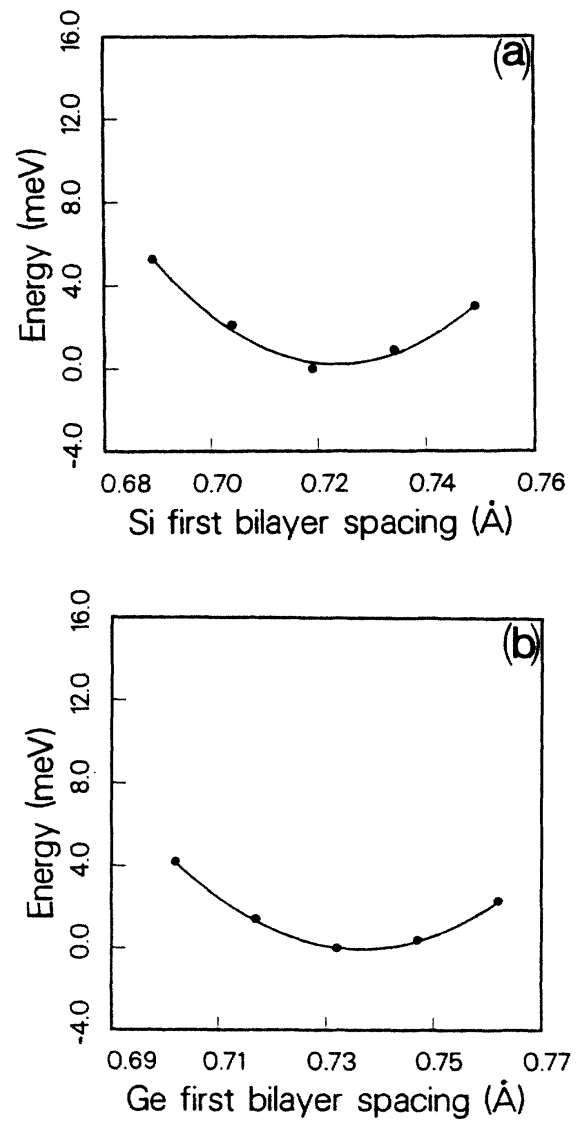

FIG. 6. Same as in Fig. 4 for the top layer vibration mode. 
TABLE IV. Comparison of calculated vibrational frequencies with experiment and with corresponding values in amorphous materials. Frequencies are in $\mathrm{meV}$. The corresponding wave number in $\mathrm{cm}^{-1}$ is also given under each frequency in brackets. $\omega_{\mathrm{H}}^{\text {stretch }}$ is the stretching mode of the hydrogen bond, $\omega_{\mathrm{H}}^{\text {wag }}$ the wagging mode of the same bond, and $\omega_{1}$ the frequency for vibration of the top layer of hydrogenated atoms.

\begin{tabular}{|c|c|c|c|c|c|c|}
\hline & Theory & $\begin{array}{c}\mathrm{Si} \\
\text { Expt. }^{\mathrm{a}}\end{array}$ & $\alpha-\mathrm{Si}: \mathbf{H}^{\mathrm{b}}$ & Theory & $\begin{array}{c}\mathrm{Ge} \\
\text { Expt. }^{\mathrm{a}}\end{array}$ & $\alpha-\mathrm{Ge}: \mathrm{H}^{\mathrm{b}}$ \\
\hline$\omega_{\mathrm{H}}^{\text {stretch }}$ & $\begin{array}{c}245 \\
(1978)\end{array}$ & $\begin{array}{c}257 \\
(2085)\end{array}$ & $\begin{array}{c}248 \\
(2000)\end{array}$ & $\begin{array}{c}231 \\
(1860)\end{array}$ & $\begin{array}{c}245 \\
(1975)\end{array}$ & $\begin{array}{c}235 \\
(1895)\end{array}$ \\
\hline$\omega_{\mathrm{H}}^{\text {wag }}$ & $\begin{array}{c}71 \\
(569)\end{array}$ & $\begin{array}{c}77 \\
(630)\end{array}$ & $\begin{array}{c}79 \\
(640)\end{array}$ & $\begin{array}{c}63 \\
(505)\end{array}$ & $\begin{array}{r}66 \\
(530)\end{array}$ & $\begin{array}{c}70 \\
(565)\end{array}$ \\
\hline$\omega_{1}$ & $\begin{array}{c}35 \\
(283)\end{array}$ & & $\begin{array}{r}26 \\
(210)\end{array}$ & $\begin{array}{c}20 \\
(162) \\
\end{array}$ & & $\begin{array}{c}15 \\
(120) \\
\end{array}$ \\
\hline
\end{tabular}

${ }^{a}$ From Refs. 11, 24, and 25.

${ }^{b}$ From Refs. 26, 27, and 28.

phous material by at least a factor of 2 . It is interesting to note that the angular force constants used by Cardo$\mathrm{na}^{32}$ to calculate the frequency $\omega_{1}$ for $\alpha$-Si:H were chosen 2.7 times smaller than values based on a fit of the elastic constants of crystalline $\mathrm{Si}^{34}$

This work was supported in part by Joint Services Electronics Program (Department of Defense) Contract
No. DAAL 03-86-K-0002. We are indebted to Dr. P. M. Marcus for introducing us to the problem, for stimulating discussions and for communicating to us experimental results prior to publication. It is a pleasure to thank $\mathrm{Dr}$. $\mathrm{K}$. C. Pandey for a critical reading of the manuscript and helpful suggestions. We thank Eugen Tarnow for interesting comments. One of us (E.K.) would like to acknowledge support by IBM.
${ }^{1}$ H. Ibach and J. E. Rowe, Surf. Sci. 43, 481 (1974).

${ }^{2}$ T. Sakurai and H. D. Hagstrum, Phys. Rev. B 12, 5349 (1975).

3J. E. Rowe, Surf. Sci. 53, 461 (1975).

4J. Appelbaum and D. R. Hamann, Phys. Rev. Lett. 34, 806 (1975).

${ }^{5}$ K. C. Pandey, T. Sakurai, and H. D. Hagstrum, Phys. Rev. Lett. 35, 1728 (1975).

${ }^{6}$ K. C. Pandey, Phys. Rev. B 14, 1557 (1976).

${ }^{7}$ K. M. Ho, M. L. Cohen, and M. Schlüter, Phys. Rev. B 15, 3888 (1977).

${ }^{8}$ K. C. Pandey, Phys. Rev. Lett. 47, 1913 (1981).

${ }^{9}$ R. D. Brigans and H. Hochst, Appl. Surf. Sci. 11-12, 368 (1982).

${ }^{10} \mathrm{H}$. Wagner, R. Butz, U. Backes, and D. Bruchmann, Solid State Commun. 38, 1155 (1981).

${ }^{11}$ H. Frotzheim, H. Lammering, and H. L. Gunter, Phys. Rev. B 27, 2278 (1983).

${ }^{12}$ K. Hermann and P. S. Bagus, Phys. Rev. B 20, 1603 (1979).

${ }^{13}$ V. Barone, F. Lelj, N. Russo, M. Toscano, F. Illas, and J. Rubio, Phys. Rev. B 34, 7203 (1986).

${ }^{14}$ G. B. Bachelet, D. R. Hamann, and M. Schlüter, Phys. Rev. B 26, 4199 (1982).

${ }^{15}$ J. Ihm, A. Zunger, and M. L. Cohen, J. Phys. C 12, 4409 (1979).

${ }^{16}$ D. M. Ceperley and B. J. Alder, Phys. Rev. Lett. 45, 566 (1980).

17J. Perdew and A. Zunger, Phys. Rev. B 23, 5048 (1984).

${ }^{18}$ P. O. Lowdin, J. Chem. Phys. 19, 1396 (1951).

${ }^{19}$ K. Rabe and J. D. Joannopoulos, Phys. Rev. B 32, 2302 (1985).
${ }^{20}$ H. J. Monkhorst an J. D. Pack, Phys. Rev. B 13, 5188 (1976),

${ }^{21}$ L. Pauling, The Nature of the Chemical Bond (Cornell University Press, Ithaca, New York, 1960).

${ }^{22}$ K. M. Ho et al. (Ref. 7) obtained a similar value (1.55 $⿱$ ) for the $\mathrm{Si}-\mathrm{H}$ bond, but considered this value to be an artifact of the limited wave-function convergence in their calculation. Our much larger basis set still gives a value larger by $\sim 4 \%$ than that of $\mathrm{Si}-\mathrm{H}$ in $\mathrm{SiH}_{4}$.

${ }^{23}$ R. Imbihl, J. E. Demuth, F. J. Himpsel, P. M. Marcus, W. A. Thompson, and F. Jona, Phys. Rev. B 36, 5037 (1987).

${ }^{24}$ M. T. Yin and M. L. Cohen, Phys. Rev. B 26, 5668 (1982).

${ }^{25}$ H. Richter, J. Trodhal, and M. Cardona, J. Non-Cryst. Solids 59-60, 181 (1983).

${ }^{26}$ For the value of $\omega_{\mathrm{H}}^{\text {wag }}$ in $\mathrm{Ge}(111): \mathrm{H}$ we took the corresponding value from $\mathrm{Ge}(100): \mathrm{H}$. This seems reasonable in light of the fact that the $\omega_{\mathrm{H}}^{\text {stretch }}$ values for the two surfaces are identical. The $\mathrm{Ge}(100): \mathrm{H}$ experimental values are from L. Papagno, X. Y. Shen, J. Anderson, G. S. Spagnolo, and G. J. Lepeyre, Phys. Rev. B 34, 7188 (1986).

${ }^{27}$ S. C. Shen, C. J. Fang, and M. Cardona, Phys. Status Solidi B 101, 451 (1980); S. C. Shen, C. J. Fang, M. Cardona, and L. Genzel, Phys. Rev. B 22, 2913 (1980).

${ }^{28}$ M. H. Brodsky, M. Cardona, and J. J. Cuomo, Phys. Rev. B 16, 3556 (1977); E. C. Freeman and W. Paul, ibid. 18, 4288 (1978); G. Lucovsky, R. J. Nemanich, and J. C. Knights, ibid. 19, 2064 (1979).

${ }^{29}$ For a review and extended references see G. Lucovsky and W. B. Pollard, The Physics of Hydrogenated Amorphous Silicon II, edited by J. D. Joannopoulos and G. Lucovsky (SpringerVerlag, Berlin, 1984). 
${ }^{30}$ C. C. Tsai and R. J. Nemanich, J. Non-Cryst. Solids 35-36, 1203 (1980).

${ }^{31}$ E. Martinez and F. Yndurain, Solid State Commun. 44, 1477 (1982).
${ }^{32}$ M. Cardona, Phys. Status Solidi B 118, 463 (1983).

${ }^{33}$ See, for example, the discussion by R. Alben, D. Wearie, J. E. Smith, Jr., and M. H. Brodsky, Phys. Rev. B 11, 2271 (1975).

${ }^{34}$ R. M. Martin, Phys. Rev. B 1, 4405 (1970). 